Man and Nature

L'homme et la nature

\title{
Le Troglodyte image fictive du Sauvage canadien
}

\section{Sante A. Viselli}

Volume 5, 1986

URI : https://id.erudit.org/iderudit/1011862ar

DOI : https://doi.org/10.7202/1011862ar

Aller au sommaire du numéro

Éditeur(s)

Canadian Society for Eighteenth-Century Studies / Société canadienne d'étude du dix-huitième siècle

ISSN

0824-3298 (imprimé)

1927-8810 (numérique)

Découvrir la revue

Citer cet article

Viselli, S. A. (1986). Le Troglodyte image fictive du Sauvage canadien. Man and Nature / L'homme et la nature, 5, 191-200. https://doi.org/10.7202/1011862ar

Copyright (c) Canadian Society for Eighteenth-Century Studies / Sociéte canadienne d'étude du dix-huitième siècle, 1986
Ce document est protégé par la loi sur le droit d'auteur. L'utilisation des services d’Érudit (y compris la reproduction) est assujettie à sa politique d'utilisation que vous pouvez consulter en ligne.

https://apropos.erudit.org/fr/usagers/politique-dutilisation/ 


\section{Le Troglodyte image fictive du Sauvage canadien}

Chaque observateur donne du Sauvage sa propre image. L'ambiguïté de cette phrase est voulue. Ce 'Seigneur' des forêts est fatalement suggestif; il vit d'une autre vie que la sienne; il vit dans l'imagination qu'il hante et qu'il féconde; il attire et il repousse. Transformé par une sorte de rêve absurde qui ne semble pas se concilier avec la logique des 'Lumières,' de ce personnage émane un attrait irrésistible qui altère le jugement de l'observateur. Bon ou mauvais Sauvage, il est assez souvent un symptôme des troubles de conscience du civilisé 'las d'être lui-même.'1

Le Sauvage est victime de l'Européen qui perçoit d'une manière fausse la réalité du monde indien. C'est un cas de projection; les signaux les plus flagrants se manifestent en linguistique: la langue du Sauvage est vue à travers la grille latine. Victime, il l'est par l'attitude du civilisé qui, dans la remise en question de son propre univers, utilise le Sauvage comme modèle naturel; dans cette confrontation l'homme qui se désigne comme civilisé raisonne en occidental. Un vide se creuse entre les deux cultures. Sans communication aujourd'hui encore ce gouffre reste à combler. Victime, il l'est du missionnaire qui ne peut que voir la méchanceté du Sauvage sans Dieu et sa bonté avec Dieu. Mais martyr, il l'est surtout par le rôle qu'on lui fait jouer dans la littérature, dans la politique et dans l'économie où il est respectivement image d'un monde utopique, enjeu politique dans les différends entre grandes puissances, et produit commercial. 
Le Sauvage, presque dépourvu de sa vie réelle, est à l'origine d'un discours justificateur des inquiétudes de l'occidental; jamais nous n'avons de témoignages authentiques relevant de la réalité indienne par l'Indien lui-même. Les choses ne changent guère et la confrontation des points de vue est donc impossible; il est évident que toutes les informations sur l'Amérique du Nord sont dialectiquement ordonnées à l'horizon d'un bien futur, façonné par et pour l'Européen. Le Sauvage est donc - comme le Troglodyte de Montesquieu - un être fictif.

Selon Jean Ehrard l'histoire édifiante des Troglodytes est une illustration de cet accord du 'raisonnement' et de la 'nature' que Montesquieu semble postuler dans l'abstrait dans l'Essai touchant les lois naturelles. ${ }^{2}$ Cet accord, produit de la fiction littéraire, touche particulièrement à une morale du sentiment retrouvée. Ces bons Troglodytes font songer au baron de La Hontan, à ses vertueux Hurons du Canada. Montesquieu se laisse ainsi prendre par l'attrait nostalgique du bon Sauvage. Dans sa polémique contre les abus de certaines institutions dégradantes pour l'homme, l'auteur des Lettres persanes fait cependant de ce dernier une figure de style. Sa démarche se caractérise par plusieurs trahisons heureuses qui montrent bien un esprit ouvert à de nouveaux horizons. Le sien n'est pas un 'bonheur de sécheresse' comme le dit Paul Hazard, mais plutôt un bonheur vécu en profondeur, présent en toute chose qu'il a analysée, expliquée, expérimentée. Son engagement n'étant pas intéressé, on voit avec plaisir l'intérêt du particulier travailler pour l'intérêt général:

Si j'avois su quelque chose qui m'eût été utile, et qui eût été préjudiciable à ma famille, je l'aurois rejeté de mon esprit; si j'avois su quelque chose, utile à ma famille, et qui ne l'eût pas été à ma patrie, j'aurois cherché à l'oublier; si j'avois su quelque chose, utile à ma patrie, et qui eût été préjudiciable à l'Europe, ou qui eût été utile à l'Europe, et préjudiciable au genre humain, je l'aurois regardé comme un crime. ${ }^{3}$

L'homme de Montesquieu, dans l'acception la plus large du mot, peut donc beaucoup donner, beaucoup enseigner, n'importe où dans l'univers, n'importe quand dans l'histoire.

Aussi a-t-on le sentiment que si le Sauvage du Canada n'apparaît pas d'une façon explicite dans les Lettres persanes No. XII, XIII et XIV, il est présent à l'esprit de l'auteur au moment de leur rédaction. Dans l'Essai touchant les lois naturelles Montesquieu fait mention du Sauvage d'Amérique à plusieurs reprises en le désignant comme 'le cannibale,' l'Américain ... libre et maître de sa conduite, il ne reconnaît aucun supérieur. ${ }^{4}$ La copie du manuscrit de cet Essai conservée aux Archives de Bordeaux présente une note ajoutée par le copiste: 'Nota.-Le manuscrit se 
termine par différents extraits des Lettres persanes, entre autres des lettres II, XII, XIII et XIV.' ${ }^{5}$ Est-ce 'l'Américain' dont il est question dans $\mathrm{l}^{\prime}$ Essai tout à fait étranger au développement de la fiction et des bienfaits qui résultent de l'observation des devoirs naturels? Bien que la fiction des Troglodytes se situe à une époque indéterminée 'dans le décor imprécis d'une Arabie de convention, ${ }^{\prime} 6$ on est porté à croire qu'elle pourrait se situer aussi bien dans un décor imprécis d'un Canada de convention. En effet les vertueux Troglodytes se caractérisent par des traits de simplicité rustique; comme les bons Sauvages, ils n'ont pas encore appris à penser. Ils possèdent les qualités innées, propres à l'Age d'or, les plus proches de la nature:

Ils avoient de l'humanité; ils connoissoient la justice; ils aimoient la vertu ... liés par la droiture du coeur ... ils voyoient la désolation générale et ne la ressentoient que par la pitié ... Ils travailloient pour l'intérêt commun ... Ils menoient une vie heureuse et tranquille ,,. Ils aimoient leurs femmes ... On alloit au Temple pour demander les faveurs des Dieux, ce n'étoit pas les richesses et une onéreuse abondance; de pareils souhaits étoient indignes des heureux Troglodytes; ils ne savoient le désirer que pour leurs compatriotes ... Le soir ils s'assembloient ... et, dans un repas frugal, ils chantoient ...

ils célébroient les grandeurs des Dieux ... ils décrivoient ensuite les délices de la vie champêtre et le bonheur d'une condition toujours parée de l'innocence ... La Nature ne fournissoit pas moins à leurs désirs qu'à leurs besoins. De ce pays heureux, la cupidité étoit étrangère; ils se faisoient des présents où celui qui donnoit croyoit toujours avoir l'avantage. Le peuple troglodyte se regardoit comme une seule famille.?

Le bon Sauvage de La Hontan se caractérise par des traits moraux assez proches de ceux du Troglodyte. Le rôle plutôt polémique que celuilà est destiné à jouer dans l'histoire des idées semble le rapprocher plus de la fausse naïveté des Persans que de la simplicité naturelle du Troglodyte; cependant la critique de ce dernier sera aussi forte que celle des Persans et plus proches du Huron. Ces deux peuples sont bons; ils sont bons parce qu'ils vivent selon les lois de la nature. Comme le Troglodyte, le Huron montre à l'humanité ses bonnes qualités:

Adario: Nous sommes nés libres et frères unis, aussi grands maîtres les uns que les autres ... Nous vivons simplement sous les lois de l'instinct et de la conduite innocente que la Nature sage nous a imprimé dès le berceau. Nous sommes tous d'accord et conformes en volonté, opinions et sentiments ... Chacun est maître de soi-même et fait tout ce qu'il veut sans rendre compte à personne et sans qu'on y 
trouve à redire ... Tu sais bien que nous et nos femmes nous nous empoisonnons le plus souvent pour nous aller tenir compagnie dans le pays des morts, lorsque l'un ou l'autre meurt. ${ }^{8}$

Haranguant le civilisé, Adario l'exhorte à revenir à cet état de nature idéal:'N'ayant ni tien ni mien, vous vivrez avec la même félicité que les Hurons. ${ }^{\prime}$ Dans un autre dialogue fictif le baron de la Hontan fait parler un médecin portugais:

Comment peut-on damner ces pauvres gens avec tant d'assurance: il est probable que leur premier Père, bien loin de pécher comme notre Adam, doit avoir eu l'âme bonne et le coeur droit, puisque ses descendants suivent exactement la loi de l'équité naturelle, et que n'admettant point de propriété de biens, de distinction ni de subordination entre eux, ils vivent comme frères, sans dispute, sans procez, sans loi et sans malice. ${ }^{10}$

Le bon Troglodyte comme le bon Sauvage se caractérise surtout par l'innocence, loin du péché d'Adam qui rend l'homme coupable et criminel même avant de naître. Le Troglodyte honore un Dieu qu'il apprend à craindre; ensuite la Religion viendra adoucir dans les moeurs 'ce que la nature y avoit laissé de trop rude. ${ }^{11}$ L'existence de Dieu est aussi présente à l'esprit du Sauvage canadien; il la sent en toute chose, ce qui fait qu'il l'adore en tout ce qui paraît au monde.'12 Ils honorent un Dieu beaucoup plus proche de celui des philosophes que de celui des dévots. La religion naturelle semble réconcilier l'homme avec lui-même, avec le monde et avec l'Etre suprême. Le Troglodyte et le Huron sont loin du pessimisme chrétien ou de la tradition sceptique de Bayle que Montesquieu refusera dans l'Esprit des lois. Les deux peuples possèdent un bonheur sans équivoques et sans compromis; leur Dieu est bien ce 'monarque' dont parle Montesquieu:'qui a plusieurs nations dans son Empire; elles viennent toutes lui porter le tribut et chacune lui parle sa langue. ${ }^{\prime 13}$ 'Et qui sçait,' dit La Hontan, 'si Dieu ne veut pas être honoré par une infinité d'hommages et de respects différens. ${ }^{\prime 14}$

Tous ces traits analogues dans les deux œuvres montrent bien un 'communisme' originel, projeté chez les Troglodytes dans un 'lointain Age d'or à jamais disparu,'et chez les Sauvages 'dans un cadre exotique en marge de la civilisation moderne. ${ }^{15}$ Ces deux mondes n'existent que dans le rêve nostalgique des deux auteurs, rêve d'un paradis perdu, d'un monde égalitaire, d'une vie innocente et vertueuse.

Pour La Hontan le rêve est, à sa façon, une expérience réellement vécue dans les forêts canadiennes avec les Sauvages. Cependant l'auteur transcende la réalité indienne et donne naissance à un mythe qui 
fécondera l'imagination d'autres philosophes, notamment celles de JeanJacques Rousseau et de Denis Diderot.

Pour Montesquieu le rêve est de courte durée. Ses Troglodytes ne relèvent pas de l'histoire, ni de l'expérience, mais de la fiction littéraire, à tendance moralisante. Il sait bien que cet 'Eldorado' n'est qu'un mirage, mais le message véhiculé est tout de même riche de bon sens. Nés à une époque où John Law bouleverse économiquement, politiquement et moralement la France, les Troglodytes montrent au monde un visage plus humain, une vie paisible dans la tranquillité et la frugalité, un retour à la terre, une pratique de la vertu, un univers où le coeur, 'ce citoyen de tous les pays, ${ }^{\prime 16}$ dicte ses lois douces dans une nature bénévole et accueillante. La cupidité et l'intérêt, les spéculations financières en Louisiane, la folle course à s'enrichir par l'achat des actions de la Compagnie des Indes ne sont que des figures de la corruption qui causa la désolation générale' des premiers Troglodytes, en l'occurrence des Français de la période du Système et qui ont voulu, au-delà des possibilités apparentes de leur nature, dépasser la réalité historique et forcer les potentialités effectives du Système même.

La petite fiction des Troglodytes, s'inscrivant dans une plus large qui est le roman, représente un macrocosme en soi dont émane une force polémique aussi considérable que la polémique soulevée par les Persans et par les Hurons de La Hontan. Cependant Montesquieu ne se borne pas seulement à la dénonciation de la corruption de son temps en idéalisant les vertus de jadis. Une fois envolé dans ce monde d'ailleurs, il ne faut pas rester prisonnier du charme de sa propre fiction; il faut revenir à la réalité historique de son époque, ce que La Hontan, par exemple, ne fera pas. Les vertueux Troglodytes ne peuvent pas subsister à l'état de nature. L'idylle n'est pas éternelle; les Troglodytes croient qu'il est temps de se choisir un roi: 'O Troglodytes! Votre vertu commence à vous peser,' dit le Vieillard en sanglotant, car dans l'état où ils se trouvent, les conditions n'étant plus les mêmes que celles de leur premier établissement, les Troglodytes doivent 'être vertueux malgré eux.'17 Vivant dans la frugalité, leurs besoins se satisfaisaient, comme ceux des Sauvages, 'aisément et également. L'égalité est donc forcée. ${ }^{18}$ Aussi, la richesse naissante pourrait-elle leur être fatale et les faire retomber dans l'anarchie et les erreurs de leurs premiers pères. Pour éviter le chaos ils auront besoin d'être gouvernés par les lois; elles rétabliront l'égalité perdue: 'Dans l'état de nature,' dit Montesquieu, les hommes naissent bien dans l'égalité; mais ils n'y sauroient rester. La société la leur fait perdre, et ils ne redeviennent égaux que par les lois. ${ }^{19}$ Désormais les moeurs ne sont guère suffisantes si on veut s'organiser en société réglée; le Vieillard troglodyte est le porteparole des convictions de Montesquieu: 
Vous aimez mieux être soumis à un prince et obéir à ses lois, moins rigides que vos moeurs. Vous savez que, pour lors, vous pourrez contenter votre ambition, acquérir des richesses, et languir dans une lâche volupté, et que, pourvu que vous évitiez de tomber dans les grands crimes, vous n'aurez pas besoin de vertu. ${ }^{20}$

Cette vertu se perd et se transforme au fur et à mesure que le peuple devient société. L'intérêt du particulier commence à s'instaurer dans les esprits et gardera les hommes dans une étroite interdépendance. Avec la perte de la frugalité naissent d'autres exigences et d'autres passions à assouvir; il faudra donc des lois pour les maîtriser et une bonne éducation pour apprendre à connaître les devoirs et pour assurer la perpétuation de la vertu, qualité naturellement intrinsèque à l'homme. Héritier de la philosophie socratique et stoïcienne selon laquelle le mal moral n'est que la conséquence de l'ignorance, l'auteur de l'Essai touchant les lois naturelles invite le lecteur à revenir à 'l'Américain dont on vient de parler'; il faudra l'éduquer en commençant par lui montrer l'inhumanité de ses actions et l'inviter à 'prendre à coeur ses véritables intérêts,' à ne pas traiter les autres d'une manière dont il n'aimerait pas être traité luimême, lui montrer par une' peinture agréable des douceurs que procure l'humanité,' les avantages qui naissent des services mutuels qu'on se rend, et la paix et la tranquillité qui en dérivent. ${ }^{21}$ Cette initiation lui inspirera des principes de prudence et non pas des devoirs:

Libre et maître de sa conduite, il ne reconnaît aucun supérieur; il faut donc lui apprendre que Dieu est le maître de tous les hommes, leur père commun, qu'il est trop sage et trop bon pour leur permettre de se déchirer les uns les autres, que d'ailleurs il a pourvu suffisamment à leur entretien... C'est ainsi que vous conduirez cet homme à la loi de la nature. ${ }^{22}$

Le Sauvage, un être aliéné dans la possession de sa liberté même, retrouve son identité d'être humain et sa raison d'être dans la reconnaissance et dans l'observation des lois de la nature. De ces lois il s'ensuit un principe fondateur de la vie en société: 'l'homme n'a pas été fait pour vivre seul.' Pour cette raison il possède les talents qui se seraient développés 'très imparfaitement, s'il passoit ses jours dans la solitude.' Il serait farouche, 'couvert de mousse,' vivant dans quelque caverne pour se protéger des intempéries, plongé dans l'oisiveté, dans la tristesse et dans l'ennui, 'errant dans les bois, tremblant au seul bruit d'une feuille, toujours dans l'appréhension des bêtes sauvages,' sans secours, en proie à la faim et à la misère. ${ }^{23}$

Ce tableau peu séduisant peint par Montesquieu n'aurait pas charmé le baron de La Hontan pour qui le fait de vivre sans 'Loix, ni juges, ni 
Prêtres,' c'est la marque même de la supériorité de l'homme de la 'Nature' sur l'homme civilisé. ${ }^{24}$ Mais les buts des deux auteurs sont différents; dans son réquisitoire contre les institutions françaises, La Hontan passe à côté de la condition réelle de l'Indien. Il lui fait parler un langage fictif qui ne lui appartient pas; Adario est le symbole de l'aliénation linguistique et culturelle dont le Sauvage en général est souvent victime. Montesquieu, pour qui le Sauvage n'est pas l'homme qui possède le mieux les lois de la nature, rapporte tout à des raisons existentielles et fonctionnelles. Il n'adopte pas le point de vue du colonisateur; il ne se fait pas non plus le champion d'un retour à la vie sauvage. Cependant, Montesquieu penche plutôt du coté de la modernité; dans l'Essai touchant les lois naturelles il dira:

Hors de la société, l'homme jouit d'une liberté qui ne sauroit lui être que fort à charge ... Dans la société chacun ne se sert de la liberté qu'autant qu'il lui en faut pour mener une vie commode et tranquille. Le mien et le tien ont leurs bornes fixes, et l'on en jouit en paix, de son droit particulier. ${ }^{25}$

On voit déjà les bons Troglodytes se choisir un roi et par cet acte ils vont se différencier de plus des Sauvages qui, eux, demeurent à un état statique. Dans ce premier état, sans connaissances et sans discipline l'homme n'a que sa propre expérience qui puisse le guider; dans la société il profite de l'expérience des autres, ce qui lui fournit de nouvelles idées. Enfin hors de la société il n'y a qu'ennui et que férocité:

La crainte ne m'abandonne jamais, tout me manque: et secours et consolation. Mais dans la société on voit régner la politesse des moeurs; Je trouve des amis qui me secourent dans le besoin, qui adoucissent mes maux, et me consolent dans la misère. ${ }^{26}$

Les paradis artificiels l'emportent donc sur les paradis naturels. Les différences très frappantes entre ces deux états dévoilent une réalité sociale qui dépasse le jeu social de l'univers du civilisé, jeu qui porte d'une critique des lois à une contestation de l'autorité, du pouvoir arbitraire, de la fausseté de certaines institutions. La réalité sociale est celle qui relève d'une législation et d'une justice qui, une fois établies, protègent les hommes du chaos. Il n'est pas question ici de savoir si la justice des lois qui régissent une société est juste; elle est nécessaire, parce qu'en dernière analyse, elle assure la paix et dans la paix chacun a le droit de jouir de son bien et de sa liberté: 'Les peuples qui vivent sous une bonne police,' dira Montesquieu dans l'Esprit des lois, 'sont plus heureux que ceux qui, sans règle et sans chefs, errent dans les forêts. ${ }^{27}$ 
Montesquieu affirme-t-il que les Sauvages sont malheureux? On pourrait, à cet égard, demeurer d'accord avec le Père Buffier quand il affirme 'que les peuples Sauvages sont aussi heureux que les peuples polis' et qu'il ne faut pas confondre les deux idées de peuples polis et de peuples heureux. ${ }^{28}$ Montesquieu, sans confondre les deux notions, invite le lecteur à placer les données par rapport au contexte dans lequel elles s'appliquent; en effet il fait une confrontation de deux mondes et de cette confrontation il résulte une différence. Il faut donc placer l'idée de bonheur ou de malheur dans cette différence et non dans l'analyse particulière de chaque monde. Le bonheur ressortira d'un principe dynamique qui conduit l'être vers l'expérience et la connaissance; même l'angoisse d'exister aura le pouvoir de changer le monde en mieux. Le malheur résultera, par contre, d'un principe statique où l'expérience et la connaissance sont réduites aux besoins physiologiques de conservation et de survivance; dans ce cas l'angoisse d'exister produira l'aliénation. Le bonheur se traduit en une métamorphose perpétuelle vers la perfection de l'être; le malheur ne peut que tendre vers l'entropie.

Maupertuis, dans son Essai de philosophie morale, fait entrer dans l'examen les plaisirs et les peines, c'est-à-dire tout ce qui est susceptible de rendre l'âme heureuse ou malheureuse. ${ }^{29} \mathrm{Il}$ ne fait point entrer,' dit Montesquieu, le bonheur de l'existence et la félicité habituelle, qui n'avertit de rien, parce qu'elle est habituelle. Nous n'appelons 'plaisir' que ce qui n'est pas habituel. ${ }^{\prime 30}$ Montesquieu ne peut pas accepter l'idée d'uniformité, car elle est en contradiction avec l'esprit même d'une nation policée, c'est-à-dire cela représenterait une espèce de retour en arrière, vers ce 'non-être' dont le philosophe avait tellement horreur:

Si nous avions continuellement le plaisir de manger avec appétit, noun n'appellerions cela un 'plaisir'; ce seroit 'existence' et 'nature.' Il ne faut pas dire que le bonheur est ce moment que nous ne voudrions pas changer pour un autre. Disons autrement: le bonheur est ce moment que nous ne voudrions pas changer pour le 'non-être. ${ }^{31}$

Le bonheur n'est pas un moment; il est une réinscription continuelle d'expériences vécues dans le temps et dans l'espace. Il est l'être, la vie même qui se déroule dans toute sa diversité.

On pourra donc tirer une conclusion; Monstesquieu ne prêche pas le retour à la nature, ni à la vie menée par les Sauvages d'Amérique. Cependant, s'il voit la façon de vivre des nations policées plus heureuse, il ne dit pas que les Sauvages sont malheureux, car-comme le rappelle Champlain-leur genre de vie est misérable 'au regard de la nôtre, mais 
heureuse entre eux qui n'ont gousté de meilleure, croyans qu'il ne s'en trouve pas de plus excellente. ${ }^{32}$

Finalement, le Sauvage-Troglodyte sort de cet Age d'or pour se moderniser, pour s'épanouir dans tous les domaines des connaissances; il s'éloignera de plus en plus de cette simplicité initiale qu'il ne pourra regagner au risque de tomber dans cette affreuse uniformité du 'non-être' qui rebutait tellement le Seigneur de la Brède. L'expérience des Troglodytes se révèle donc positive avec ouverture sur l'avenir; par contre, les Sauvages-ceux de La Hontan en particulier-demeurant statiques et attachés à l'uniformité de leur existence se révèlent comme une expérience négative. Montesquieu ne verra dans leur état que la négation même de la vie: 'Sauvages,' dit-il: 'hommes et non pas citoyens. Ils respiroient l'air et ne vivoient pas. ${ }^{\prime 3}$

\section{SANTE A. VISELLI}

Université Memorial

\section{Notes}

1 Paul Hazard, La Pensée européenne au XVIII siècle, de Montesquieu à Lessing (Paris: éd. Boivin 1946), I, 124

2 Jean Ehrard, L'Idée de nature en France dans la première moitié du XVIII siècle (Chambéry: Imprimeries réunies 1963), I, 347

3 Montesquieu, Histoire véritable, Oeuvres complètes de Montesquieu, éd. Roger Caillois (Paris: Gallimard 1979), I, 462.

4 Montesquieu, Essai touchant les loix naturelles, Oeuvres complètes de Montesquieu, éd. André Masson (Paris: Nagel 1955), III, 186-7

5 Xavier Vedère, Introd., Essai touchant les loix naturelles, Oeuvres complètes de Montesquieu, éd. A. Masson III, 175

6 Jean Ehrard, L'Idée de nature en France ..., I, 348

7 Montesquieu, Lettres persanes, XII, Oeuvres complètes de Montesquieu, éd. R. Caillois, I, 149-50

8 La Hontan, Dialogues avec un Sauvage (Paris: éd. sociales 1973) 93-117

9 Ibid., 124

10 La Hontan, Nouveaux voyages de $M^{r}$ le Baron de Lahontan dans l'Amérique septentrionale (La Haye: Frères l'Honoré 1703) 253. Il faut cependant remarquer que Montesquieu, à la fin de la lettre XII déjà citée, laisse entendre que les 
Troglodytes ont-même si d'une façon très vague-l'idée de propriété privée: 'Le peuple troglodyte se regardoit comme une seule famille: les troupeaux étoient presque toujours confondus; la seule peine qu'on s'épargnoit ordinairement, c'étoit de les partager.'

11 Montesquieu, Lettres persanes, XII, éd. R. Caillois, 149

12 La Hontan, Mémoires de l'Amérique septentrionale ou la suite des Voyages de $M^{r}$ le Baron de Lahontan (La Haye: Frères l'Honoré 1703), 113

13 Montesquieu, Pensées, 1454, Oeuvres complètes de Montesquieu, éd. André Masson (Paris: Nagel 1950) II, 420-1

14 La Hontan, Nouveaux voyages ... 254

15 Jean Ehrard, L'Idée de nature en France ..., II, 524

16 Montesquieu, Lettre persanes, LXVII, éd. R. Caillois, I, 228

17 Ibid., XIV, 153

18 Montesquieu, Esprit des lois, XVIII, 17 Oeuvres complètes de Montesquieu, éd. R. Caillois (Paris: Gallimard 1976), II, 540

19 Ibid., VIII, 3, 352

20 Montesquieu, Lettres persanes, XIV, éd. R. Caillois, I, 153

21 Montesquieu, Essai touchant les lois naturelles et la distinction du juste et de linjuste, Oeuvres complètes de Montesquieu (Paris: Nagel 1955), III, 187.

22 Ibid., 188

23 Ibid., 195-196

24 La Hontan, Mémoires ..., 102

25 Montesquieu, Essai touchant les lois naturelles ..., éd. A. Masson, III, 196

26 Ibid., 196

27 Montesquieu, Esprit des lois, V, 11, éd. R. Caillois, II, 291

28 Le Père Buffier, Cours de sciences sur des principes nouveaux et simples pour former le langage, l'esprit et le coeur, dans l'usage ordinaire de la vie (Paris: Cavalier et Giffart 1732) 974

29 Pierre Louis Moreau de Maupertuis, Essai de philosophie morale (Berlin: S. éd., 1749), 54-74

30 Montesquieu, Pensées, 2010, Oeuvres complètes de Montesquieu, éd. A. Masson II, 620

31 Ibid., 2010, 621

32 Samuel Champlain, Voyages du Sieur de Champlain, ou Journal ès découvertes de la Nouvelle-France (Paris: s. éd. 1830), I, 374

33 Montesquieu, Pensées, 1555, Oeuvres complètes de Montesquieu, éd. A. Masson, II, 449 